\title{
DYNAMICALLY CONVEX FINSLER METRICS AND $J$-HOLOMORPHIC EMBEDDING OF ASYMPTOTIC CYLINDERS
}

\author{
ADAM HARRIS AND GABRIEL P. PATERNAIN
}

\begin{abstract}
We explore the relationship between contact forms on $\mathbb{S}^{3}$ defined by Finsler metrics on $\mathbb{S}^{2}$ and the theory developed by H. Hofer, K. Wysocki and E. Zehnder in [9, 10. We show that a Finsler metric on $\mathbb{S}^{2}$ with curvature $K \geq 1$ and with all geodesic loops of length $>\pi$ is dynamically convex and hence it has either two or infinitely many closed geodesics. We also explain how to explicitly construct $J$-holomorphic embeddings of cylinders asymptotic to Reeb orbits of contact structures arising from Finsler metrics on $\mathbb{S}^{2}$ with $K=1$ thus complementing the results obtained in 8 .
\end{abstract}

\section{INTRODUCTION}

A contact form $\lambda$ on a closed, connected, oriented manifold of odd dimension is said to be dynamically convex if the Conley-Zehnder index of any contractible periodic orbit of the Reeb vector field is at least three. This notion generalises the case of convex hypersurfaces in $\mathbb{R}^{4}$ on which the contact form is simply the restriction of the standard one-form $\lambda_{0}$ from the ambient space. When the hypersurface is $\mathbb{S}^{3}$, the Reeb flow induces a fibration by orbits of constant period, namely the Hopf fibration. If the hypersurface is an ellipsoid $\mathbb{E}_{p, q}$ defined by the equation $p|z|^{2}+q|w|^{2}=1\left(z, w\right.$ denoting coordinates of $\left.\mathbb{C}^{2}\right)$ then an interesting dichotomy arises between the dynamics of the Reeb flow for $\frac{p}{q}$ rational or irrational. In the rational case there are still infinitely many periodic orbits, though not all are of the same minimal period, while in the irrational case there are just two periodic orbits. This dichotomy extends to any strictly convex hypersurface, since it was shown by Hofer, Wysocki and Zehnder [9] that the Reeb vector field $X$ of any contact form satisfying the dynamic convexity condition on the three-sphere has either two or infinitely many periodic orbits. A central role in their theory is played by the existence of finite-energy

2000 Mathematics Subject Classification. 32Q65, 53D10, 58B20. 
$J$-holomorphic embeddings of the cylinder in the "symplectisation" of a contact manifold, from which a periodic orbit is realised as the asymptotic limit of an end of the embedded cylinder.

For the standard example associated with the Hopf fibration on $\mathbb{S}^{3}$ there is a direct correspondence between the asymptotic limit of finiteenergy $J$-holomorphic embeddings in the symplectisation on $\mathbb{S}^{3} \times \mathbb{R}$ and germs of plane algebroid curves centred at the origin of a sufficiently small ball in $\mathbb{C}^{2}$, via their "link" with the boundary-sphere. It may then be asked whether a more general correspondence can be established between plane algebroid curve-germs and $J$-holomorphic embeddings in the symplectisation of other contact forms $\lambda$ on $\mathbb{S}^{3}$. It should be noted that a similar question has been studied extensively in the context of pseudoholomorphic curves in a symplectic manifold (cf., e.g., [17]) though the question there is local rather than asymptotic in nature. In [8] it was shown that near periodic orbits of "elliptic type" (cf. locally recurrent orbits, [8]) such that the partial almost complex endomorphism $j$, compatible with the contact structure, is Reeb-invariant (i.e., the Lie derivative $\mathcal{L}_{X} j=0$ ), finite-energy $J$-holomorphic embeddings $\psi$ of the cylinder may be represented holomorphically in a suitable tubular coordinate neighbourhood of the orbit. After transformation of the cylinder to a punctured disc $D \backslash\{0\}$ via the choice of a complex coordinate $z$, the periodic component of $\psi$ naturally subdivides $D$ into "quasi-sectors", the number of these being determined by the topological degree of $\psi$ restricted to the circle $|z|=c$, as $c$ approaches zero (i.e., as the axial coordinate of the cylinder approaches infinity), and being referred to as the "charge" of the mapping. In the direction spanned by a disc $\Delta$, transversal to the orbit, $\psi$ is represented by holomorphic functions defined on each of the quasi-sectors of $D$, which are hinged together along common edges by the return map $\alpha$, induced on $\Delta$ by the Reeb flow. With respect to $(\lambda, j)$ satisfying the above criteria and for a given charge $n$, each finite-energy $J$ - holomorphic embedding of the cylinder in $\mathbb{S}^{3} \times \mathbb{R}$ therefore gives rise to a collection of $n$ holomorphic functions, whose continuity at adjacent boundaries of their domains is mediated by $\alpha$ (cf. [8, Theorem 1]).

For any contact structure $(\lambda, j)$ such that $\mathcal{L}_{X} j=0$ and $\alpha$ is the identity (e.g., the standard $\lambda_{0}$ restricted to $\mathbb{S}^{3}$ ) it is possible to move explicitly back and forth between finite-energy $J$-holomorphic embeddings of the cylinder in the tubular neighbourhood of a periodic orbit and algebraic curve-germs at the origin in $\mathbb{C}^{2}$ of the form $\left(z^{n}, F(z)\right)$. A similarly explicit correspondence was obtained in [8] for contact structures on $\mathbb{S}^{3}$ diffeomorphically equivalent to the restriction of $\lambda_{0}$ to a rational ellipsoid (on which $\alpha$ is a non-trivial rational rotation near 
each of two exceptional orbits). Implicit in these examples is the fact that the contact form has rotational symmetry along the axis of a suitable tubular coordinate neighbourhood of the orbit, thus allowing the map $\psi$ conversely to be determined (up to local diffeomorphic equivalence) by the charge $n$ and holomorphic functions $F_{k}$, predefined on formal sectors $Q_{k}$, such that $F_{k+1}=\alpha \circ F_{k}$ at the common boundary of adjacent sectors, $0 \leq k \leq n-1$ (cf. Theorem A below). As we note in the present article, this crucial symmetry property is also held (up to local gauge correction) by any contact form for which $\alpha$ is an irrational rotation (as in the case of the irrational ellipsoids), so that a $\psi$ of charge $n$ is again determined by the holomorphic data $F_{k}$ :

Theorem A. Suppose, for the ensemble $(M, \lambda, j)$ that $\mathcal{L}_{X} j=0$ and the return map $\alpha$ induced locally by the Reeb flow near a locally recurrent orbit $\mathcal{P}$ corresponds to an irrational rotation. For positive integer $n$ let $Q_{k}$ denote the formal sectors of a disc $D \subset \mathbb{C}$ defined by

$$
2 \pi \frac{k}{n}<\arg (z)<2 \pi \frac{k+1}{n}, \quad 0 \leq k \leq n-1,
$$

with holomorphic functions $w=F_{k}(z)$ defined on $Q_{k}$ such that

$$
\left.F_{k}\right|_{\bar{Q}_{k} \cap \bar{Q}_{k+1}}=\left.\alpha \circ F_{k+1}\right|_{\bar{Q}_{k} \cap \bar{Q}_{k+1}}, \quad \lim _{z \rightarrow 0} F_{k}(z)=0
$$

for each $k$. Modulo a local gauge correction of the form

$$
\hat{\lambda}=\lambda-d f
$$

for some smooth function $f$, defined in a tubular neighbourhood of $\mathcal{P}$ such that $X_{\lambda}(f)=0$, these holomorphic data determine a finite-energy $J$-holomorphic curve of charge $n$, asymptotic to $\mathcal{P}$.

But to how large a class of contact structures on $\mathbb{S}^{3}$ do these correspondences apply? More broadly, to how large a class of structures do the dynamic convexity results of Hofer, Wysocki and Zehnder apply? An important class of examples of triples $(M, \lambda, j)$ is given by the contact form and almost complex endomorphism $j$ naturally induced on the three-manifold corresponding to the unit tangent bundle of a Riemann surface $\Sigma$ by the geodesic flow of a metric $g$ on $\Sigma$ (cf., e.g., [13]). If the additional Lie symmetry condition $\mathcal{L}_{X} j=0$ is assumed then $g$ must have Gaussian curvature $K \equiv 1$ and up to isometry we just have $\Sigma=\mathbb{S}^{2}$. But the class of required structures is much broader if, instead of a Riemannian metric, one considers a Finsler structure on the two-sphere, i.e., a hypersurface $M \subset T \mathbb{S}^{2}$ and a surjective submersion $\pi: M \rightarrow \mathbb{S}^{2}$ such that for all $p \in \mathbb{S}^{2}$ the fibre $\pi^{-1}(p)$ is a smooth, closed strictly convex curve enclosing the origin in $T_{p} \mathbb{S}^{2}$ (cf. section two). 
The associated structure equations derived from a canonical framing of $T^{*} M$ depend on three functions $I, J, K$ over $M$. When the Finsler structure corresponds specifically to a Riemannian metric, it follows that $I \equiv 0$ and $K$ corresponds to the pullback of Gaussian curvature from $\Sigma$. The induced almost complex endomorphism $j$ on a Finsler contact manifold $(M, \lambda)$ satisfies $\mathcal{L}_{X} j=0$ precisely when $K \equiv 1$ for any $I, J$ (cf. Lemma 3.1).

A famous class of contact structures due to A. Katok [11] arises from convex hypersurfaces $M \subset T \mathbb{S}^{2}$ on which $K \equiv 1$ and the return map $\alpha$ associated with the Reeb flow near either of its two distinguished periodic orbits is a rotation. The Finsler structures connected with these examples incorporate what are known as Randers metrics, coming from special perturbations of the norm derived from a Riemannian metric. When a Finsler structure on $\Sigma$ with $K \equiv 1$ admits a non-trivial Killing field, one may perturb to a family of non-Randers examples with the same properties, as is shown in section 3.2, following independent observations of P. Foulon and W. Ziller [18]. All the hypersurfaces in question are doubly covered by $\mathbb{S}^{3}$, such that the contact forms lift to tight contact structures on the three-sphere. In sections three and four a precise description of this lifting reveals that the Katok examples correspond exactly with those induced by the restriction of $\lambda_{0}$ to $\mathbb{E}_{p, q} \subset \mathbb{C}^{2}$. It is then worth noting that the non-Randers structures of section 3.2 lie genuinely beyond the standard class of examples coming from convex hypersurfaces of $\mathbb{R}^{4}$. Returning to the broader notion of dynamic convexity for contact structures on $\mathbb{S}^{3}$, it is asked in the final section of this article whether a corresponding criterion can be found in terms of Finsler structures on $\mathbb{S}^{2}$. Theorem B provides this criterion for a given Finsler structure, via a lower bound on the length $\ell$ of its shortest geodesic loop (we shall say that the Finsler metric is dynamically convex if its associated contact form is dynamically convex):

Theorem B. Let $F$ be a Finsler metric on $\mathbb{S}^{2}$ such that $K \geq \delta>0$. If $\ell>\pi / \sqrt{\delta}$, then $F$ is dynamically convex.

In particular, by the results in [9] any such Finsler metric has either two or infinitely many closed geodesics. We note that recently, V. Bangert and Y. Long [1] have shown that any Finsler metric on $\mathbb{S}^{2}$ has two closed geodesics.

Acknowledgements: The authors would like to express their warm thanks to Z. Shen for his helpful communications during their research 
towards this article. We are also very grateful to V. Bangert and H.B. Rademacher for their comments and remarks.

\section{J-HOLOMORPHIC CYLINDERS NEAR LOCALLY RECURRENT ORBITS OF A CONTACT THREE-MANIFOLD}

Let $M$ denote a compact, oriented three-manifold with contact form $\lambda$ and associated plane-field $\xi \subset T M$ corresponding to $\operatorname{ker}(\lambda)$. Let $X_{\lambda}$ denote the Reeb vector field associated with this structure on $M$, together with an almost complex structure $J$ acting on $\xi$ such that the symmetric tensor defined by $\left.d \lambda(*, J *)\right|_{\xi}$ is positive definite. Consider a periodic orbit of the Reeb flow, denoted $\mathcal{P}$, and a tubular neighbourhood $T_{\mathcal{P}} \subset M$. If $\Delta$ represents a disc centred at the origin in $\mathbb{R}^{2}$, let $\tilde{\Delta} \subset M$ be an embedded image such that the origin is mapped to the unique element $p_{0}$ of $\mathcal{P} \cap \tilde{\Delta}$, with $\tilde{\Delta}$ itself corresponding to a transverse slice of $T_{\mathcal{P}}$. The Reeb flow will be assumed moreover to be Lyapunov-stable near $\mathcal{P}$ in the sense that for all $p \in \tilde{\Delta}^{\prime}$, where $\Delta^{\prime} \subseteq \Delta$ is a sufficiently small disc centred at the origin, there exists a unique solution $\gamma_{p}:[0, \infty) \rightarrow M$ to the equation

$$
\frac{d \gamma_{p}}{d t}=X_{\lambda}\left(\gamma_{p}(t)\right), \quad \gamma_{p}(0)=p
$$

which depends smoothly on both $t$ and $p$, and remains inside $T_{\mathcal{P}}$ for all $t \geq 0$. Given $p \in \tilde{\Delta}^{\prime}$, we will define $(i) \tau(p)$ to be the smallest $t>0$ such that $\gamma_{p}(t) \in \tilde{\Delta}$, (ii) $\Gamma_{p}:=\gamma_{p}((0, \tau(p)])$ and for each connected open neighbourhood of the origin, $\Omega \subseteq \tilde{\Delta}$,

$$
\text { (iii) } \Gamma(\Omega):=\cup_{p \in \Omega} \Gamma_{p} \text {. }
$$

We may now consider a recursively defined system of neighbourhoods $\left\{\Omega_{k}\right\}$, such that $\Omega_{0}:=\tilde{\Delta}^{\prime}$, while $\Omega_{k}$ denotes the origin-component of $\Gamma\left(\Omega_{k-1}\right) \cap \Omega_{k-1}$. The set $\Omega_{\infty}:=\cap_{k=0}^{\infty} \Omega_{k}$ was seen in [8] proposition 1 to be conformally equivalent to a disc whenever it corresponds to an open subset of $\tilde{\Delta}^{\prime}$. As in [8], the Reeb flow will be said to be "locally recurrent" near a periodic orbit $\mathcal{P}$ if it is Lyapunov-stable within a tubular neighbourhood $T_{\mathcal{P}}$ and for any sufficiently small embedded disc $\tilde{\Delta}$, corresponding to a transversal slice through $T_{\mathcal{P}}$ at some point $p_{0}$, the limit set $\Omega_{\infty} \subseteq \tilde{\Delta}^{\prime} \subseteq \tilde{\Delta}$ is open. An orbit $\mathcal{P}$ itself may also be referred to as "locally recurrent" in this context. In passing we note that if the orbit is elliptic of twist type, then by Moser's twist map theorem it will be locally recurrent. Moreover, the local recurrence implies that the characteristic multipliers are unimodular. 
Under the assumption that the Reeb flow is locally recurrent near $\mathcal{P}$, we now select $\Omega_{\infty} \times\left\{\vartheta_{0}\right\}$ as coordinate disc within the initial Martinet tube (in which $\lambda=f \cdot(d \vartheta+x d y)$, where $\vartheta$ denotes the periodic coordinate and $f$ denotes a function such that $f(0,0, \vartheta) \equiv \tau_{0}$ and $\nabla f(0,0, \vartheta) \equiv \mathbf{0}$, though these facts are not used here). Without loss of generality, let $\vartheta_{0}$ be zero and consider the cylinder $\Omega_{\infty} \times[0,2 \pi]$, which maps to the tube via the obvious identification $\bmod (2 \pi)$. The cylinder has $\mathcal{P}$ as its axis, $x=y=0$, and the Reeb vector field in Martinet coordinates already looks like $\frac{1}{\tau_{0}} \frac{\partial}{\partial \vartheta}$ when restricted to $\mathcal{P}$. There is no consequent loss of generality if we "normalise" $\lambda$ by the constant multiple $\frac{1}{\tau_{0}}$, so that the minimal period is effectively 1 . In particular this will ensure that the notion of "charge" for a $J$-holomorphic mapping $\psi$ of the punctured disc, as introduced below, is consistent with the standard definition corresponding to the limit of the integral of $\psi^{*} \lambda$ over the circle $|z|=c$ as $c$ approaches zero (and is specifically an integer). By analogy with the standard construction of Darboux coordinates, the next step is to define

$$
\mathcal{C}:=\left\{(p, t) \mid p=(x, y) \in \Omega_{\infty}, \quad 0 \leq t \leq \tau(p)\right\},
$$

and a homeomorphism

$$
h: \mathcal{C} \rightarrow \Omega_{\infty} \times[0,2 \pi],\left.\quad h\right|_{\Omega_{\infty} \times\{0\}}=1,
$$

which is smooth for all $0<t<\tau(p)$, coming from solutions of the ordinary differential equation

$$
\frac{d \gamma_{p}}{d t}=X_{\lambda}\left(\gamma_{p}(t)\right)
$$

It follows that on the interior of $\mathcal{C}$, the standard contact form $\lambda_{0}$ and $\lambda^{\prime}:=h^{*} \lambda$ have the same Reeb vector field, corresponding to $\frac{\partial}{\partial t}$. We now consider the Cauchy-Riemann system

$$
\begin{gathered}
\pi\left(\left(h^{-1} \psi\right)_{\eta}\right)+J \pi\left(\left(h^{-1} \psi\right)_{\zeta}\right)=0 \quad(*), \\
\lambda^{\prime}\left(\left(h^{-1} \psi\right)_{\zeta}\right)=-a_{\eta}, \quad \lambda^{\prime}\left(\left(h^{-1} \psi\right)_{\eta}\right)=a_{\zeta} \quad(\dagger),
\end{gathered}
$$

satisfied by some finite-energy $J$-holomorphic map $\psi$ of a punctured neighbourhood $D \backslash\{0\} \subset \mathbb{C}$ into the Martinet tubular neighbourhood of $\mathcal{P}(\times \mathbb{R})$. For a sufficiently "thin" neighbourhood of $\mathcal{P}$, the standard projection $\left(v_{1}, v_{2}, v_{3}\right) \mapsto\left(v_{1}, v_{2}\right)$ determines a linear isomorphism $\mu$ between $\xi^{\prime}:=\operatorname{ker}\left(\lambda^{\prime}\right)$ and $\mathbb{R}^{2}$. Hence we define a $2 \times 2$ matrix-valued function $j(x, y)=\mu \circ J \circ \mu^{-1}$, such that $\mathbf{x}:=(x, y)$ implies $\left(^{*}\right)$ can be written in the form

$$
\mathbf{x}_{\eta}(z)+j \mathbf{x}_{\zeta}(z)=0
$$

Let $\alpha$ denote the diffeomorphism of $\Omega_{\infty} \times\{0\}$ defined by the return map $\alpha(p):=\gamma_{p}(\tau(p))$, hence $\alpha(0)=0$. As seen in [8], if $\mathcal{L}_{X_{\lambda}} J=0$, 
then in a neighbourhood of $0 \in \Delta$, the smooth automorphism $\alpha$ is equivalent to a rotation, via a diffeomorphism $\varphi: \Delta^{\prime \prime} \rightarrow U \subseteq \Omega_{\infty}$ such that $\varphi_{*}^{-1} \circ j \circ \varphi_{*}=j_{0}$ (the standard multiplication by i). More specifically, let $\Omega_{\infty}^{\prime}$ denote the simply connected domain inside $U$ which is stabilised by the Reeb flow. The diffeomorphism $\hat{\alpha}:=\varphi^{-1} \circ \alpha \circ \varphi$ then acts on $\varphi^{-1}\left(\Omega_{\infty}^{\prime}\right) \subseteq \Delta^{\prime \prime}$ as an automorphism such that $\hat{\alpha}(0)=0$ under the assumption of local recurrence. The additional assumption $\mathcal{L}_{X_{\lambda}} J=0$ implies that $\alpha^{*} j=j$, hence in particular $\hat{\alpha} j_{0}=j_{0} \hat{\alpha}$, i.e., $\hat{\alpha}$ is a conformal automorphism. Modulo a conformal transformation identifying $\varphi^{-1}\left(\Omega_{\infty}^{\prime}\right)$ with a disc, $\hat{\alpha}$ is then equivalent to a rotation.

Now $\tilde{\mathbf{x}}_{\zeta}:=\varphi_{*}^{-1}\left(\mathbf{x}_{\zeta}\right)$ and $\tilde{\mathbf{x}}_{\eta}:=\varphi_{*}^{-1}\left(\mathbf{x}_{\eta}\right)$ implies

$$
\tilde{\mathbf{x}}_{\eta}(\eta, \zeta)+j_{0} \cdot \tilde{\mathbf{x}}_{\zeta}(\eta, \zeta)=\mathbf{0}(\dagger *) .
$$

As described in [8], each "branch" of

$$
\Psi:=(h \circ(\varphi \times 1))^{-1} \psi
$$

is defined smoothly in the interior and continuously up to the boundaries of a quasi-sector $Q_{k}$ in $D \backslash\{0\}$, with discontinuities arising at points $z_{0}$ lying on the smooth arcs, corresponding to $\vartheta^{-1}(0)$, that bound adjacent sectors (in the usual way " \pm " will be used to denote opposite sides of the boundary). Discontinuities of the transverse projection of $\Psi$ are therefore described by the relations

$$
\lim _{z \rightarrow z_{0}^{ \pm}} \tilde{\mathbf{x}}(z):=\tilde{\mathbf{x}}^{ \pm}\left(z_{0}\right) \Rightarrow \hat{\alpha}\left(\tilde{\mathbf{x}}^{-}\left(z_{0}\right)\right)=\tilde{\mathbf{x}}^{+}\left(z_{0}\right) \text {. }
$$

Hence on each $Q_{k} \subset D \backslash\{0\},(\dagger *)$ defines a holomorphic function $w=F_{k}(z)$ which partially describes a branch of $\Psi$, such that

$$
\left.F_{k}\right|_{\bar{Q}_{k} \cap \bar{Q}_{k+1}}=\left.\hat{\alpha} \circ F_{k+1}\right|_{\bar{Q}_{k} \cap \bar{Q}_{k+1}}, \quad 0 \leq k \leq n-1,
$$

where $n$ denotes the charge (or asymptotic degree) of $\psi$ in relation to the periodic orbit $\mathcal{P}$.

Returning now to the particular form of the equations $(\dagger)$ in $\mathcal{C}$ note that any $\lambda^{\prime}$ with $\frac{\partial}{\partial t}$ as its Reeb vector field must take the general form

$$
\lambda^{\prime}=d t+f_{1}(\mathbf{x}) d x+f_{2}(\mathbf{x}) d y .
$$

Such a local presentation of the contact form allows decoupling of $(\dagger)$ into an inhomogeneous Cauchy-Riemann equation. This property is preserved under the diffeomorphism $\varphi$, however, if it is assumed that $\mathcal{L}_{X_{\lambda}} J=0$, hence in particular the matrix $j$ above is independent of $t$. In this case, letting $\Re$ and $\Im$ signify real and imaginary parts, and $u=t+\mathbf{i} a,(\dagger)$ becomes

$$
\frac{\partial}{\partial \bar{z}}\left[\left.u\right|_{Q_{k}}\right]=-\left\{f_{1}\left(F_{k}(z)\right) \frac{\partial \Re\left(F_{k}\right)}{\partial \bar{z}}+f_{2}\left(F_{k}(z)\right) \frac{\partial \Im\left(F_{k}\right)}{\partial \bar{z}}\right\}
$$




$$
=-\frac{1}{2}\left[\left(f_{1}+\mathbf{i} f_{2}\right) \circ F_{k}(z)\right] \cdot \overline{F_{k}^{\prime}(z)},
$$

keeping in mind that this equation is defined smoothly only on the interior of each quasi-sector $Q_{k}$. Define $\omega:=\frac{1}{2}\left(f_{1}+\mathbf{i} f_{2}\right)(w, \bar{w}) d \bar{w}$, so that

$$
\begin{gathered}
\lambda^{\prime}=d t+2 \Re(\omega), \text { and } \\
\frac{1}{2}\left[\left(f_{1}+\mathbf{i} f_{2}\right) \circ F_{k}(z)\right] \cdot \overline{F_{k}^{\prime}(z)} d \bar{z}=F_{k}^{*} \omega .
\end{gathered}
$$

Now $\mathcal{L}_{X_{\lambda}} \lambda=0$ implies $\hat{\alpha}^{*} \Re(\omega)=\Re(\omega)$. In particular, f $:=\left(f_{1}, f_{2}\right)$ implies $\Re(\omega)=(\mathbf{f}, *)$ with respect to the standard inner product on $\mathbb{R}^{2}$, and hence $\hat{\alpha}_{*}^{t} \mathbf{f}=\mathbf{f}$. Similarly $\Im(\omega)=\left(j_{0} \mathbf{f}, *\right)$, while $\mathcal{L}_{X_{\lambda}} J=0$ implies $\hat{\alpha}_{*}^{t} j_{0}=j_{0} \hat{\alpha}_{*}^{t}$, so that

$$
\hat{\alpha}^{*} \Im(\omega)=\left(\hat{\alpha}_{*}^{t} j_{0} \mathbf{f}, *\right)=\left(j_{0} \mathbf{f}, *\right)=\Im(\omega) .
$$

It follows that $\hat{\alpha}^{*} \omega=\omega$, and hence

$$
\begin{gathered}
\left.F_{k}^{*} \omega\right|_{\bar{Q}_{k} \cap \bar{Q}_{k+1}}=\left.F_{k}^{*}\left(\hat{\alpha}^{*} \omega\right)\right|_{\bar{Q}_{k} \cap \bar{Q}_{k+1}}=\left.\left(\hat{\alpha} \circ F_{k}\right)^{*} \omega\right|_{\bar{Q}_{k} \cap \bar{Q}_{k+1}} \\
=\left.F_{k+1}^{*} \omega\right|_{\bar{Q}_{k} \cap \bar{Q}_{k+1}} .
\end{gathered}
$$

There now exists a continuous function $G(z, \bar{z})$ on $D$ such that

$$
\left.G(z, \bar{z}) d \bar{z}\right|_{Q_{k}}:=F_{k}^{*} \omega, \quad 0 \leq k \leq n-1,
$$

which is moreover continuously differentiable (cf. [8]), and hence

$$
\hat{G}(z, \bar{z}):=\int_{D} \frac{G(\mu, \bar{\mu})}{\mu-z} d \mu \wedge d \bar{\mu}
$$

is a twice-continuously differentiable function on $D$. Moreover, the inhomogeneous equation satisfied by $u$ now has the form

$$
\frac{\partial}{\partial \bar{z}}\left[\left.u\right|_{Q_{k}}\right]=G, \quad 0 \leq k \leq n-1
$$

and with a little additional argument we have

Theorem $2.1([8])$. Let $(\psi, a): D \backslash\{0\} \rightarrow M \times \mathbb{R}$ be a J-holomorphic curve of finite energy and charge $n$ at $z=0$, asymptotic to a locally recurrent periodic orbit $\mathcal{P}$, near which $\mathcal{L}_{X_{\lambda}} J=0$. Consider any tubular neighbourhood of $\mathcal{P}$ in $M$, diffeomorphic to $\Delta \times \mathbb{S}^{1}$ such that $\{0\} \times \mathbb{S}^{1} \approx$ $\mathcal{P}$. There exists a diffeomorphic change of coordinates in $\Delta \times[0,2 \pi)$ such that on each quasi-sector $Q_{k} \subset D \backslash\{0\}$ the map $(\psi, a)$ can be expressed in the form

$$
\left(F_{k}(z), H_{k}(z)-\frac{1}{2 \pi \mathbf{i}} \hat{G}(z, \bar{z})\right), \quad 0 \leq k \leq n-1,
$$

where $F_{k}, H_{k}$ are holomorphic on $Q_{k}$ and continuous on $\bar{Q}_{k}$, such that

$$
\left.F_{k}\right|_{\bar{Q}_{k} \cap \bar{Q}_{k+1}}=\left.\hat{\alpha} \circ F_{k+1}\right|_{\bar{Q}_{k} \cap \bar{Q}_{k+1}},
$$


while each $H_{k}$ corresponds to an analytic branch of $\frac{1}{2 \pi \mathbf{i}} \log (\rho), \operatorname{ord} d_{0}(\rho)=$ $n$. Moreover, the function $\hat{G}$ belongs to $C^{2}(D)$ and is bounded by $K|z|$. Finally, if $\alpha=1$, then each $F_{k}$ is the restriction of a single function $F$ holomorphic on $D, F(0)=0$.

Conversely, given an ensemble $(M, \lambda, J), \mathcal{L}_{X_{\lambda}} J=0$, with locally recurrent periodic orbit $\mathcal{P}$ corresponding to the asymptotic limit of some $J$-holomorphically embedded cylinder of finite energy, we may ask for the essential holomorphic data which determine such $J$-holomorphic curves in general near $\mathcal{P}$. Our main result in this direction is the following:

Theorem A. Suppose, for the ensemble $(M, \lambda, J)$ above, that the return map $\alpha$ induced locally by the Reeb flow near a recurrent orbit $\mathcal{P}$ corresponds to an irrational rotation. For positive integer $n$ let $Q_{k}$ denote the formal sectors of a disc $D \subset \mathbb{C}$ defined by

$$
2 \pi \frac{k}{n}<\arg (z)<2 \pi \frac{k+1}{n} \quad 0 \leq k \leq n-1,
$$

with holomorphic functions $w=F_{k}(z)$ defined on $Q_{k}$ such that

$$
\left.F_{k}\right|_{\bar{Q}_{k} \cap \bar{Q}_{k+1}}=\left.\hat{\alpha} \circ F_{k+1}\right|_{\bar{Q}_{k} \cap \bar{Q}_{k+1}}, \lim _{z \rightarrow 0} F_{k}(z)=0
$$

for each $k$. Modulo a local gauge correction of the form

$$
\hat{\lambda}=\lambda-d f,
$$

for a smooth function $f$, defined in a tubular neighbourhood of $\mathcal{P}$ such that $X_{\lambda}(f)=0$, these holomorphic data determine a finite-energy $J-$ holomorphic curve of charge $n$, asymptotic to $\mathcal{P}$.

Proof. Returning to the general form of $\lambda$ with respect to the coordinate tube $\mathcal{C}$ above, we have

$$
\lambda^{\prime}=d t+f_{1}(\mathbf{x}) d x+f_{2}(\mathbf{x}) d y=d t+\Re(\omega),
$$

noting that $\hat{\alpha}^{*} \Re(\omega)=\Re(\omega)$, in the case of $\alpha$ an irrational rotation (hence in particular the orbit of any point $w$ under $\alpha$ is dense in a circle of radius $|w|)$. Now

$$
\Re(\omega)=\eta(\rho) d \nu+\zeta(\rho) d \rho=\eta(|w|)(x d y-y d x)+d f,
$$

where $w=x+\mathbf{i} y, \rho=|w|, \nu=\arg (w)$, and

$$
f(\rho)=\int_{0}^{\rho} \zeta(r) d r .
$$


Letting $\hat{\eta}\left(\rho^{2}\right)=\eta(\rho)$, and after making the gauge correction $\hat{\lambda}:=$ $\lambda-d f:=d t+\Re(\hat{\omega})$, we have

$$
\hat{\omega}=\hat{\eta}(w \bar{w}) \cdot \mathbf{i} w d \bar{w},
$$

and hence

$$
\Phi\left(|w|^{2}\right):=\int_{0}^{|w|^{2}} \hat{\eta}(r) d r
$$

implies $\hat{\omega}=\mathbf{i} \bar{\partial} \Phi\left(|w|^{2}\right)$. It should be noted at once that $d \hat{\lambda}=d \lambda, X_{\lambda}(f)=$ 0 together imply that $X_{\lambda}$ remains the Reeb vector field of the contact form defined by $\hat{\lambda}$. Recall that

$$
\left.F_{k}\right|_{\bar{Q}_{k} \cap \bar{Q}_{k+1}}=\left.\hat{\alpha} \circ F_{k+1}\right|_{\bar{Q}_{k} \cap \bar{Q}_{k+1}}, \lim _{z \rightarrow 0} F_{k}(z)=0
$$

indicates that there exists a single smooth function $|F|$ on $D$, corresponding to $F_{k}$ on each $Q_{k}$, hence we may define

$$
G(z, \bar{z}):=\mathbf{i} \bar{\partial} \Phi\left(|F|^{2}\right) \text {, and } \hat{G}(z, \bar{z}):=2 \pi \mathbf{i} \cdot \mathbf{i} \Phi\left(|F|^{2}\right)=-2 \pi \Phi\left(|F|^{2}\right) \text {. }
$$

Under the assumption that $\mathcal{P}$ is already the asymptotic limit of some finite energy mapping of the punctured disc (or cylinder), we recall moreover from the discussion of [8, that the return time $\tau(p)$ for each $p \in \Omega_{\infty}^{\prime}$ is constant (i.e., normalised to value 1 ). Note that the rotational symmetry of the contact form ultimately implies that $\hat{G}$ is a real-valued function, compatible with the formal specification of simple sectors $Q_{k}$ as domains of the holomorphic functions $F_{k}$, and moreover that the $t$-component of a $J$-holomorphic mapping derived from these data can be defined by $t=\frac{1}{2 \pi} \arg \left(z^{n}\right)$. Now from [8], Theorem 1, we may write

$$
u=t+\mathbf{i} a=\frac{1}{2 \pi \mathbf{i}}\left(\log \left(z^{n}\right)+2 \pi \Phi\left(|F|^{2}\right)\right),
$$

from which it follows that the associated $a$-component must be

$$
a(z)=\frac{-1}{2 \pi} \log \left(|z|^{n}\right)-\Phi\left(|F|^{2}\right) .
$$

One or two remarks should be made concerning the almost complex structure with respect to which the mapping $\Psi$, determined sector-wise by the $F_{k}(z)$ and the corresponding analytic branches of $\frac{1}{2 \pi \mathbf{i}}\left(\log \left(z^{n}\right)+\right.$ $2 \pi \Phi\left(|F|^{2}\right)$ ), may be said to represent a $J$-holomorphic mapping of charge $n$ of the cylinder into $\mathcal{T}_{\mathcal{P}} \times \mathbb{R}$, for a tubular neighbourhood $\mathcal{T}_{\mathcal{P}}$. It is straightforward to see that in a sufficiently thin tube, the standard projection mapping defines an isomorphism between $\mathbb{R}^{2}$ and the contact planes of both $\lambda$ and $\hat{\lambda}$ at any given point which we will denote by $\beta: \xi \rightarrow \hat{\xi}$, i.e., $\beta(\mathbf{v})=\mathbf{v}-\hat{\lambda}(\mathbf{v}) X$, where $X$ denotes the common Reeb vector field of $\lambda$ and $\hat{\lambda}$. The almost complex structure 
$\hat{J}:=\beta \circ J \circ \beta^{-1}$ is then automatically induced on $\operatorname{ker}(\hat{\lambda})$. Moreover, given $\mathbf{v}^{\prime} \in \operatorname{ker}(\hat{\lambda})$, we have

$$
\begin{aligned}
& d \hat{\lambda}\left(\mathbf{v}^{\prime}, \hat{J} \cdot \mathbf{v}^{\prime}\right)=d \hat{\lambda}(\beta(\mathbf{v}), \hat{J} \cdot \beta(\mathbf{v}))=d \hat{\lambda}(\beta(\mathbf{v}), \beta(J \cdot \mathbf{v})) \\
& =d \hat{\lambda}(\mathbf{v}-\hat{\lambda}(\mathbf{v}) X, J \cdot \mathbf{v}-\hat{\lambda}(J \cdot \mathbf{v}) X)=d \lambda(\mathbf{v}, J \cdot \mathbf{v}) .
\end{aligned}
$$

Hence the quadratic form $\left.d \hat{\lambda}(*, \hat{J} *)\right|_{\hat{\xi}}$ is also positive definite.

It remains now to check that the pseudoholomorphic curve $\Psi$, defined with respect to $\hat{\lambda}, \hat{J}$ in $\mathcal{T}_{\mathcal{P}} \times \mathbb{R}$ is of finite energy. Following [9], let $\mathcal{F}$ denote the space of smooth functions $h: \mathbb{R} \rightarrow[0,1]$ such that $h^{\prime} \geq 0$, and define extensions $\hat{\lambda}_{h}$ of the contact form from $\mathcal{T}_{\mathcal{P}}$ to $\mathcal{T}_{\mathcal{P}} \times \mathbb{R}$ such that $\hat{\lambda}_{h}(p, a):=h(a) \cdot \hat{\lambda}(p)$. The "energy" of $\Psi$ is then defined as

$$
E(\Psi):=\sup _{\mathcal{F}} \int_{D \backslash\{0\}} \Psi^{*} d \hat{\lambda}_{h}
$$

Clearly,

$$
\begin{gathered}
\int_{D \backslash\{0\}} \Psi^{*} d \hat{\lambda}_{h}=\int_{|z|=1} \Psi^{*} \hat{\lambda}_{h}-\lim _{\varepsilon \rightarrow 0} \int_{|z|=\varepsilon} \Psi^{*} \hat{\lambda}_{h} \\
=\int_{|z|=1} h(a) \Psi^{*} \hat{\lambda}-\lim _{\varepsilon \rightarrow 0} \int_{|z|=\varepsilon} h(a) \Psi^{*} \hat{\lambda} .
\end{gathered}
$$

Now

$$
\Psi^{*} \hat{\lambda}=-\eta(|F|) d \nu(F)+\frac{n}{2 \pi} d \arg (z),
$$

where the formula

$$
\nu=\arg (w)=\tan ^{-1}\left(-\mathbf{i} \frac{w-\bar{w}}{w+\bar{w}}\right)
$$

implies

$$
d \nu(F)=2 \Re\left(\frac{d \nu}{d w} \cdot F^{\prime}(z) d z\right)=\Re\left(\frac{-\mathbf{i} F^{\prime}}{F} d z\right) .
$$

(Here we have adopted a harmless abuse of notation, in the sense that the ratios $\frac{F_{k}^{\prime}}{F_{k}}$ define a single continuous function on $D \backslash\{0\}$ when $\alpha$ is a rotation). Note, inside any $|z|=\varepsilon$ sufficiently small, that $z=0$ is the unique zero of the smooth function $|F(z)|$, and hence

$$
\left|\int_{|z|=\varepsilon} \Psi^{*} \hat{\lambda}-n\right| \leq \int_{|z|=\varepsilon} \frac{|\eta(|F|)|\left|F^{\prime}\right|}{|F|} \cdot \varepsilon \cdot d \arg (z),
$$

where the latter quantity approaches zero as $\varepsilon$ goes to zero, if it is recalled that $\eta(\rho)$ is a smooth function such that $\eta(0)=0$. Now $|z| \rightarrow 0$ implies $a \rightarrow+\infty$, and thus

$$
E(\Psi)=\sup _{\mathcal{F}}\left(\int_{|z|=1} h\left(-\Phi\left(|F(z)|^{2}\right)\right) \cdot \Psi^{*} \hat{\lambda}-n \cdot h(+\infty)\right),
$$


which is clearly finite.

In the next section we will consider a class of examples of contact structures to which the above theorem may be applied, but it should first be estalished that holomorphic data of the sort specified are in plentiful supply. For arbitrary $n$, consider $f(z)$ holomorphic on $D \subset \mathbb{C}$, such that $\operatorname{ord}_{0}(f)>n$, and an arbitrary irrational rotation of the form $\alpha=e^{2 \pi \mathbf{i} c}, 0<c<1$. Let $D$ be divided into formal sectors as in theorem 1, with

$$
F_{0}(z):=z^{-n c} f(z), F_{k+1}(z)=\alpha \cdot F_{k}(z), 0 \leq k \leq n-1,
$$

noting that $F_{n}(z)=F_{0}(z)$. For convenience we may define $z^{-n c}=$ $e^{-n c \log (z)}$, where $\log (z)$ denotes an analytic branch of the complex logarithm defined on $\mathbb{C}$ minus the positive real axis.

\section{EXAMPLES}

3.1. Canonical coframing. Let $\Sigma$ be a closed oriented connected surface. A smooth Finsler structure on $\Sigma$ is a smooth hypersurface $M \subset T \Sigma$ for which the canonical projection $\pi: M \rightarrow \Sigma$ is a surjective submersion having the property that for each $x \in \Sigma$, the $\pi$-fibre $\pi^{-1}(x)=M \cap T_{x} \Sigma$ is a smooth, closed, strictly convex curve enclosing the origin $0_{x} \in T_{x} \Sigma$.

Given such a structure it is possible to define a canonical coframing $\left(\omega_{1}, \omega_{2}, \omega_{3}\right)$ on $M$ that satisfies the following structural equations (see [2, Chapter 4]):

$$
\begin{aligned}
& d \omega_{1}=-\omega_{2} \wedge \omega_{3}, \\
& d \omega_{2}=-\omega_{3} \wedge\left(\omega_{1}-I \omega_{2}\right), \\
& d \omega_{3}=-\left(K \omega_{1}-J \omega_{3}\right) \wedge \omega_{2} .
\end{aligned}
$$

where $I, K$ and $J$ are smooth functions on $M$. The function $I$ is called the main scalar of the structure and it vanishes if and only if $M$ is the unit circle bundle of a Riemannian metric. When $I=0$, i.e. when the Finsler structure is Riemannian, $K$ is the $\pi$-pullback of the Gaussian curvature.

Let $X_{1}, X_{2}$ and $X_{3}$ be the vector fields on $M$ that are dual to the coframing $\left(\omega_{1}, \omega_{2}, \omega_{3}\right)$. The form $\omega_{1}$ is the canonical contact form of $M$ whose Reeb vector field is the geodesic vector field $X_{1}$.

As a consequence of (1) 3 3 ) the framing $\left(X_{1}, X_{2}, X_{3}\right)$ satisfies the commutation relations:

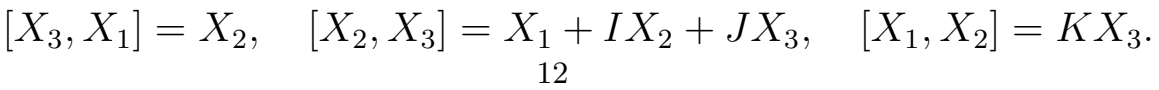


Note that the coframing also defines a natural almost complex structure $\mathbb{J}$ on $\xi=\operatorname{ker} \omega_{1}$. Indeed, we may set:

$$
\mathbb{J}\left(x X_{2}+y X_{3}\right)=y X_{2}-x X_{3} .
$$

If we let $\eta=x X_{2}+y X_{3} \in \operatorname{ker} \omega_{1}$ then using (11) we see that:

$$
d \omega_{1}(\eta, \Xi \eta)=x^{2}+y^{2}
$$

and thus $\mathbb{J}$ is compatible with the contact structure.

Lemma 3.1. $\mathcal{L}_{X_{1}} \mathbb{J}=0$ if and only if $K=1$.

Proof. Let $\phi_{t}$ be the flow of $X_{1}$. Note that $\mathcal{L}_{X_{1}} \mathbb{J}=0$ if and only if $d \phi_{t}$ is an isometry of the inner product in $\operatorname{ker} \omega_{1}$, given $d \omega_{1}(*, \mathbb{J} *)$. (Recall that $\phi_{t}$ preserves $d \omega_{1}$.)

Let $\eta=x X_{2}+y X_{3} \in \operatorname{ker} \omega_{1}$ and write:

$$
d \phi_{t}(\eta)=x(t) X_{2}+y(t) X_{3}
$$

Thus the flow $\phi_{t}$ is an isometry of the inner product $d \omega_{1}(*, \mathbb{J} *)$ if and only if

$$
\frac{d}{d t}\left(x(t)^{2}+y(t)^{2}\right)=0
$$

equivalently if and only if

$$
x \dot{x}+y \dot{y}=0 .
$$

Write

$$
\eta=x(t) d \phi_{-t}\left(X_{2}\right)+y(t) d \phi_{-t}\left(X_{3}\right)
$$

and differentiate with respect to $t$ to obtain

$$
0=\dot{x} X_{2}+x\left[X_{1}, X_{2}\right]+\dot{y} X_{3}+y\left[X_{1}, X_{3}\right] .
$$

Using the structure equations (4) and regrouping we have:

$$
0=(\dot{x}-y) X_{2}+(\dot{y}+x K) X_{3},
$$

hence

$$
\begin{gathered}
\dot{x}=y, \\
\dot{y}+K x=0 .
\end{gathered}
$$

If we use the last two equations in (5) we see that $\phi_{t}$ is an isometry if and only if

$$
x y(1-K)=0
$$

and thus $\mathcal{L}_{X_{1}} \mathbb{J}=0$ if and only if $K=1$. 
Remark 3.1. Note that the proof above shows that in general, if we let $\eta \in \operatorname{ker} \omega_{1}$ and write

$$
d \phi_{t}(\eta)=x(t) X_{2}+y(t) X_{3}
$$

then

$$
\begin{gathered}
\dot{x}=y, \\
\dot{y}+K x=0 .
\end{gathered}
$$

We will use this fact later on.

We now recall some global consequences of $K=1$ as explained by $\mathrm{R}$. Bryant in [6]. The first thing to observe is that the structure equations imply:

$$
\begin{aligned}
& \phi_{t}^{*} \omega_{1}=\omega_{1} \\
& \phi_{t}^{*} \omega_{2}=\cos t \omega_{2}+\sin t \omega_{3} \\
& \phi_{t}^{*} \omega_{3}=-\sin t \omega_{2}+\cos t \omega_{3}
\end{aligned}
$$

Suppose that $\Sigma$ is geodesically complete and connected. Then it can be shown that $\Sigma$ is diffeomorphic to $S^{2}$ and there exists a unique orientation reversing isometry $A: \Sigma \rightarrow \Sigma$ such that $\left.d A\right|_{M}=\phi_{\pi}$ (we call $A$ a quasi-antipodal map). Moreover for any point $p \in \Sigma$ every unit speed geodesic leaving $p$ passes through $A(p)$ at distance $\pi$ and $\Sigma$ has diameter $\pi$. According to [6, Proposition 4] we have the following dichotomy:

(1) $A^{2}$ is the identity on $\Sigma$ in which case all geodesics are closed with the same minimal period $2 \pi$;

(2) $A^{2}$ has exactly two fixed points, say $\sigma$ and $A(\sigma)$. Moreover there exists a positive definite inner product on $T_{\sigma} \Sigma$ that is preserved by $d\left(A^{2}\right)(\sigma): T_{\sigma} \Sigma \rightarrow T_{\sigma} \Sigma$ and there is an angle $\theta \in(0,2 \pi)$ such that $d\left(A^{2}\right)(\sigma)$ is counterclockwise rotation by $\theta$ in this inner product.

Here we will be mostly interested in case (2). We note that this case has two possible subcases. Suppose that $\theta / 2 \pi$ is rational and write $\theta=2 \pi p / q$ where $0<p \leq q$ with $p$ and $q$ coprime. Then $A^{2 q}$ is the identity and thus $\phi_{2 \pi q}=$ identity, that is, every orbit of $\phi$ is closed with period $2 \pi q$, although some orbits may have smaller minimal period.

When $\theta / 2 \pi$ is irrational, then the iterates of $A^{2}$ are dense in a circle of isometries of the Finsler surface $\Sigma$. This circle of isometries fixes $\sigma$ and $A(\sigma)$ and $\Sigma$ is rotationally symmetric about $\sigma$. Hence the geodesic flow of $\Sigma$ is completely integrable with a "Clairaut" first integral. The surface is also symmetric with respect to $A$ about a circle (the equator) 
$E$. The unit tangent vectors to $E$ determine two closed orbits $\gamma_{ \pm}$. The equator divides $\Sigma$ into two disks $D_{\sigma}$ and $D_{A(\sigma)}$ which contain $\sigma$ and $A(\sigma)$ respectively. ( $A$ maps $D_{\sigma}$ to $D_{A(\sigma)}$ and fixes $E$ setwise.)

Let $S$ be the subset of $M$ given by those pairs $(x, v)$ where $x \in E$ and $v$ points inside the region $D_{\sigma}$. The set $S$ (diffeomorphic to $E \times(0, \pi)$ ) is a section of the geodesic flow with return map $\left.\phi_{2 \pi}\right|_{S}=\left.d\left(A^{2}\right)\right|_{S}$. We see that there are no other closed orbits besides $\gamma_{ \pm}$.

If $\pi \circ \gamma_{+}$is the closed geodesic which travel around $E$ counterclockwise (as seen from $\sigma$ ) then we find the lengths of $\pi \circ \gamma_{ \pm}$to be:

$$
\begin{aligned}
& \ell_{+}:=\ell\left(\pi \circ \gamma_{+}\right)=2 \pi-d\left(p, A^{2}(p)\right), \\
& \ell_{-}:=\ell\left(\pi \circ \gamma_{-}\right)=2 \pi+d\left(A^{2}(p), p\right),
\end{aligned}
$$

where $p$ is any point in $E$. Since $\theta / 2 \pi$ is irrational we see that $\ell_{ \pm} / 2 \pi$ are irrational and in view of (7) and (8) we conclude that $\gamma_{ \pm}$are elliptic orbits of $\phi$. It is also clear that they are locally recurrent.

3.2. Examples with only two closed geodesics. Summarizing the discussion above, Theorem A can be applied to a Finsler metric on $\mathbb{S}^{2}$ with $K=1$ and $\theta / 2 \pi$ irrational. Examples of such metrics are given by the well known Katok examples [11] analyzed by W. Ziller in [18]. The fact that these metrics have $K=1$ is proved by Z. Shen [16] (see also [14, Section 5] for a discussion of these examples).

However these are not the only examples and a Katok type construction also gives a larger class as we now explain.

Suppose $F$ is a Finsler metric on $\mathbb{S}^{2}$ with $K=1$ and $A^{2}=I d$ (i.e. all the geodesics are closed and with the same minimal period). Suppose in addition that $F$ admits a nontrivial Killing field $V$. We may suppose without loss of generality that the flow $f_{t}$ of $V$ is such that $f_{2 \pi}=I d$.

Define a 1-parameter family of Finsler metrics $G_{\varepsilon}$ ( $\varepsilon$ small) by giving its co-metric $G_{\varepsilon}^{*}$ in $T^{*} \mathbb{S}^{2}$ as follows:

$$
G_{\varepsilon}^{*}(x, p)=F^{*}(x, p)+\varepsilon p(V(x)) .
$$

An unpublished result of P. Foulon asserts that $G_{\varepsilon}$ also has $K=1$ (this can be checked along the lines of the calculations in [18]). The Katok examples arise when $F$ is the standard Riemannian metric with $K=1$ in $\mathbb{S}^{2}$.

We now note that Bryant 4, 5, has produced several families of Finsler metrics with $K=1$ and $A^{2}=I d$. Among them there are subfamilies with rotational symmetry which are not of Randers type. For example Theorem 10 in 4 gives a 1-parameter family of rotationally invariant projectively flat Finsler metrics with $K=1$. 
To complete our construction we note that Ziller in [18] observed that for a Finsler metric defined by (9) with $\varepsilon$ irrational, the only closed geodesics of $G_{\varepsilon}$ are those invariant under $f_{t}$. Hence there will be only two closed geodesics for $\varepsilon$ irrational.

We remark that the results in [3] show that the Katok examples are the only examples (up to isometry, of course) of Randers metrics with $K=1$. A Randers metric is a Finsler metric of the form $\sqrt{g_{x}(v, v)}+$ $\theta_{x}(v)$, where $g$ is a Riemannian metric and $\theta$ is a 1 -form.

\section{Lifting Finsler metrics to CONTACT STRUCTURES ON $\mathbb{S}^{3}$}

Let $F$ be a Finsler metric on $\mathbb{S}^{2}$. The Lagrangian $\frac{1}{2} F^{2}$ gives rise to a Legendre transform $\ell_{F}: T \mathbb{S}^{2} \backslash\{0\} \rightarrow T^{*} \mathbb{S}^{2} \backslash\{0\}$ and if we let $\lambda$ be the Liouville 1 -form on $T^{*} \mathbb{S}^{2}$, it is well known that $\ell_{F}^{*} \lambda$ restricted to the unit sphere bundle $M$ of $F$ is precisely the contact form $\omega_{1}$ from the previous section.

On $T^{*} \mathbb{S}^{2}$ we also have a Finsler co-metric $F^{*}$ such that $F=F^{*} \circ \ell_{F}$ and a corresponding unit co-sphere bundle $M^{*}$. We consider on $\mathbb{S}^{2}$ the canonical metric with curvature 1 . It has an associated Legendre tranform $\ell_{0}: T \mathbb{S}^{2} \rightarrow T^{*} \mathbb{S}^{2}$. In what follows $|\cdot|$ denotes the norm of the canonical metric in both $T \mathbb{S}^{2}$ and $T^{*} \mathbb{S}^{2}$.

Observe that there exists a unique smooth function $g: T^{*} \mathbb{S}^{2} \backslash\{0\} \rightarrow$ $\mathbb{R}^{+}$such that $F^{*}(x, p)=g(x, p)|p|$. ( $g$ is homogeneous of degree zero in $p$.)

Let $r: M_{0}^{*} \rightarrow M^{*}$ be given by

$$
r(x, p)=(x, p / g(x, p)) .
$$

We identify $\mathbb{S}^{3}$ with $\mathbb{S U}(2)$ and $\mathbb{S}^{2}$ with the matrices in $\mathbb{S U}(2)$ of the form

$$
x=\left(\begin{array}{cc}
i t & z \\
-\bar{z} & -i t
\end{array}\right),
$$

where $t \in \mathbb{R}, z \in \mathbb{C}$ and $t^{2}+|z|^{2}=1$. If $A \in \mathbb{S U}(2)=\mathbb{S}^{3}$ and $x \in \mathbb{S}^{2}$ is as above then $A^{-1} x A \in \mathbb{S}^{2}$. This also gives a natural embedding of $\mathbb{S}^{2}$ in $\mathbb{R}^{3}$. Below we will often write $(t, z)$ to indicate a point in $\mathbb{S}^{2}$ instead of the corresponding matrix in $\mathbb{S U}(2)$.

Consider the matrices:

$$
j=\left(\begin{array}{cc}
0 & 1 \\
-1 & 0
\end{array}\right) \in \mathbb{S}^{2}, \quad k=\left(\begin{array}{cc}
0 & i \\
i & 0
\end{array}\right) \in \mathbb{S}^{2} .
$$

Now let $\mathbb{G}: \mathbb{S}^{3} \rightarrow M_{0}$ be the following map:

$$
\mathbb{G}(A)=\left(A_{16}^{-1} j A, A^{-1} k A\right)
$$


It is easy to see that the unit vectors $A^{-1} j A, A^{-1} k A$ in $\mathbb{R}^{3}$ are orthogonal. Let $A \in \mathbb{S U}(2)$ be written as:

$$
A=\left(\begin{array}{cc}
w_{1} & w_{2} \\
-\bar{w}_{2} & \bar{w}_{1}
\end{array}\right)
$$

where $\left(w_{1}, w_{2}\right) \in \mathbb{C}^{2}$ and $\left|w_{1}\right|^{2}+\left|w_{2}\right|^{2}=1$. The map $\mathbb{G}$ may also be written as

$$
\mathbb{G}(A)=\left[\left(2 \Im\left(w_{1} w_{2}\right), \bar{w}_{1}^{2}+w_{2}^{2}\right),\left(-2 \Re\left(w_{1} w_{2}\right), i\left(\bar{w}_{1}^{2}-w_{2}^{2}\right)\right)\right] .
$$

If $\lambda_{0}$ is the canonical contact form on $\mathbb{S}^{3}\left(\lambda_{0}(x)=\langle i x, *\rangle\right)$ and $\omega_{1}^{0}$ is the contact form of $M_{0}$, then it is not hard to check that (and it is explicitly done in [7, Proposition 1.1]):

$$
\mathbb{G}^{*} \omega_{1}^{0}=2 \lambda_{0} .
$$

We now claim (compare with [7, Proposition 1.1]):

Lemma 4.1. Let $f:=1 / g \circ \ell_{0}: T \mathbb{S}^{2} \backslash\{0\} \rightarrow \mathbb{R}^{+}$. Then

$$
\mathbb{G}^{*} \ell_{0}^{*} r^{*}\left(\left.\lambda\right|_{M^{*}}\right)=2(f \circ \mathbb{G}) \lambda_{0} \text {. }
$$

Proof. Let $\tau: T^{*} \mathbb{S}^{2} \rightarrow \mathbb{S}^{2}$ be the canonical projection and note that

$$
r^{*} \lambda_{(x, p)}(\xi)=p / g(d \tau(d r(\xi)))=\frac{1}{g} \lambda_{(x, p)}(d \tau(\xi))
$$

and that

$$
\ell_{0}^{*}(\lambda / g)=\omega_{0}^{1} /\left(g \circ \ell_{0}\right)
$$

Combining these two equalities with (11) the lemma follows.

The lemma is saying that the geodesic flow of a Finsler metric on $\mathbb{S}^{2}$ is (up to a double covering) smoothly conjugate to the Reeb flow of a (tight) contact form on $\mathbb{S}^{3}$ of the form $h \lambda_{0}$ where $h=2(f \circ \mathbb{G})$ and $f$ is related to the Finsler metric as described in the lemma. Conversely, if we have a contact form $h \lambda_{0}$ with $h(-A)=h(A)$, then $h$ will give rise to functions $f$ and $g$ as above. In general, for an arbitrary $h$ invariant under the antipodal map, the hypersurface $M^{*}$ of $T^{*} \mathbb{S}^{2}$ determined by $g$ does not need to be fibrewise strictly convex, but it will be clearly starshaped. Note that in the proof of the lemma we did not really need $M^{*}$ to come from a Finsler metric. If $M^{*}$ is just starshaped, then $\left.\lambda\right|_{M^{*}}$ is also a contact form.

Summarizing, the lemma gives this: there is a 1-1 correspondence between starshaped hypersurfaces of $T^{*} \mathbb{S}^{2}$ and smooth positive functions $h$ on $\mathbb{S}^{3}$ with $h(A)=h(-A)$. If in addition $M^{*}$ is fibrewise strictly convex we obtain a Finsler metric. 


\section{Ellipsoids And the KatoK EXAmples}

In contact geometry there is a well studied class of examples given by the ellipsoids

$$
\mathbb{E}_{p, q}:=\left\{\left(w_{1}, w_{2}\right) \in \mathbb{C}^{2}: p\left|w_{1}\right|^{2}+q\left|w_{2}\right|^{2}=1\right\},
$$

where $p$ and $q$ are positive real numbers. The restriction of $\lambda_{0}$ to $\mathbb{E}_{p, q}$ determines a Reeb flow whose dynamics is very simple: the flow is just $\phi_{t}\left(w_{1}, w_{2}\right)=\left(w_{1} e^{i p t}, w_{2} e^{i q t}\right)$. There are two periodic orbits corresponding to $w_{1}=0$ and $w_{2}=0$. These are the only periodic orbits if $p / q$ is irrational, whereas $\mathbb{E}_{p, q}$ is foliated by periodic orbits if $p / q$ is rational (but not all will have the same minimal period).

On the other hand, in Finsler geometry there are the well known Katok examples, which in particular provide examples of Finsler metrics with only two closed geodesics. The Finsler co-metric of the Katok examples in geodesic polar coordinates $(r, \phi) \in(0, \pi) \times[0,2 \pi]$ is

$$
F_{\varepsilon}^{*}\left(r, \phi, p_{r}, p_{\phi}\right)=\sqrt{p_{r}^{2}+\frac{1}{\sin ^{2} r} p_{\phi}^{2}}+\varepsilon p_{\phi},
$$

where $\varepsilon \in(-1,1)$. We will show in this section that the ellipsoids and the Katok examples are related precisely by the correspondence described in the previous section.

Let $h: \mathbb{S}^{3} \rightarrow \mathbb{R}^{+}$be the function

$$
h\left(w_{1}, w_{2}\right)=\frac{1}{p\left|w_{1}\right|^{2}+q\left|w_{2}\right|^{2}} .
$$

Also let $\varphi: \mathbb{S}^{3} \rightarrow \mathbb{E}_{p, q}$ be given by $\varphi\left(w_{1}, w_{2}\right)=\sqrt{h\left(w_{1}, w_{2}\right)}\left(w_{1}, w_{2}\right)$. It is easy to check that

$$
\varphi^{*} \lambda_{0}=h \lambda_{0}
$$

and hence we can think of the Reeb flow of the ellipsoids as being defined on $\mathbb{S}^{3}$ with contact form $h \lambda_{0}$ where $h$ is given by (13). Clearly $h(A)=h(-A)$. To find $f$ we write $\mathbb{G}(A)=(x, v) \in M_{0}$ where $x=(t, z)$ and $v=(b, \eta)$. The expressions for $t, z, b, \eta$ are given by (10). Using them we derive

$$
\begin{aligned}
& \bar{w}_{1}^{2}=\frac{z-i \eta}{2}, \\
& w_{2}^{2}=\frac{z+i \eta}{2} .
\end{aligned}
$$

Using that $\left(w_{1}, w_{2}\right) \in \mathbb{S}^{3}$ we obtain

$$
f(x, v)=\frac{1}{(p-q)|z-i \eta|+2 q} .
$$


We now introduce geodesic polar coordinates $(r, \phi)$ on $\mathbb{S}^{2}$ such that

$$
\begin{aligned}
& t=\cos r, \\
& z=\sin r e^{i \phi} .
\end{aligned}
$$

Thus $(b, \eta)=(\dot{t}, \dot{z})$. In these coordinates the Legendre transform $\ell_{0}$ is simply:

$$
p_{r}=\dot{r}, \quad p_{\phi}=\dot{\phi} \sin ^{2} r .
$$

We can now compute $g=1 /\left(f \circ \ell_{0}^{-1}\right)$ in $\left(r, \phi, p_{r}, p_{\phi}\right)$-coordinates:

$$
\begin{aligned}
g\left(r, \phi, p_{r}, p_{\phi}\right) & =2 q+(p-q) \sqrt{\dot{r}^{2} \cos ^{2} r+\sin ^{2} r(1+\dot{\phi})^{2}} \\
& =2 q+(p-q) \sqrt{p_{r}^{2} \cos ^{2} r+\sin ^{2} r+2 p_{\phi}+\frac{p_{\phi}^{2}}{\sin ^{2} r}} .
\end{aligned}
$$

This is the expression of $g$ on $M_{0}^{*}$, that is, when in addition

$$
p_{r}^{2}+\frac{p_{\phi}^{2}}{\sin ^{2} r}=1
$$

Thus if we simplify it further we obtain

$$
g\left(r, \phi, p_{r}, p_{\phi}\right)=2 q+(p-q)\left(1+p_{\phi}\right)=p+q+(p-q) p_{\phi} .
$$

But in view of (12) the value of $F_{\varepsilon}^{*}$ on $M_{0}^{*}$ is just $1+\varepsilon p_{\phi}$. Note that by homogeneity a Finsler metric is completely determined by its value on $M_{0}^{*}$. Hence if we choose $p$ and $q$ such that $p+q=1$, the ellipsoids induce, under the correspondence described in the previous section, exactly the Katok examples with $\varepsilon=p-q$.

Remark 5.1. In [9, Theorem 1.1] the authors show that a strictly convex hypersurface $S \subset \mathbb{R}^{4}$ carries either 2 or infinitely many periodic orbits. The ellipsoids, of course, provide examples of this dichotomy and the authors point out that it is not true that the first alternative holds only for the irrational ellipsoids. They also remark in [9, Page 200] that M. Herman constructed examples of hypersurfaces $S$ which are $C^{\infty}$-close to the ellipsoid, admit only two periodic orbits but have a transitive flow on $S$. Apparently these examples of Herman are unpublished, but we remark here that it is easy to construct such examples using the results above combined with Katok's main result in [11]. Indeed, Katok shows in [11] that given any $r$, one can approximate $F_{\varepsilon}$ in the $C^{r}$ topology by a Finsler metric $F$ with ergodic geodesic flow and only two closed geodesics. We have shown above that under the lifting procedure described in Lemma 4.1, $F_{\varepsilon}$ lifts to an ellipsoid, and hence $F$ will give rise to a smooth hypersurface $S$ which is $C^{r}$-close to 
an ellipsoid and such that the flow on $S$ has only two closed orbits and is transitive.

Note that the examples described in Subsection 3.2 give rise to a new 1-parameter family of hypersurfaces in $\mathbb{R}^{4}$ exhibiting the same dynamics and dichotomy as the ellipsoids.

Finally we note that if under the lifting described in Lemma $4.1 \mathrm{a}$ Finsler metric gives rise to a strictly convex hypersurface, then it will carry either 2 or infinitely many closed geodesics. Recently, V. Bangert and Y. Long [1] have shown that any Finsler metric on $\mathbb{S}^{2}$ has two closed geodesics. In the next section we will describe geometric conditions on the Finsler metric that ensure dynamical convexity so the main results in [9] can be applied.

\section{Dynamically COnvex Finsler metrics}

We first recall the definition of dynamically convex contact form $\lambda$ on a closed, connected and oriented manifold $M$ with $\pi_{2}(M)=0$.

The Reeb vector field $X$ is transversal to the contact structure $\xi=$ ker $\lambda$ so that naturally we have a splitting $T_{x} M=\mathbb{R} X(x) \oplus \xi_{x}$. Let $\phi_{t}$ be the flow of $X$. Clearly $d \phi_{t}(x): \xi_{x} \rightarrow \xi_{\phi_{t}(x)}$ is symplectic with respect to $d \lambda$. A contractible periodic solution $x$ with period $T$ has an integer-valued index $\mu(x, T)$ which we briefly recall (cf. [9]).

Let $D \subset \mathbb{C}$ be the closed unit disk. Choose a smooth map $\sigma$ : $D \rightarrow M$ such that $\sigma\left(e^{2 \pi \mathbf{i} t / T}\right)=x(t)$. Choose a symplectic trivialization $\psi: \sigma^{*} \xi \rightarrow D \times \mathbb{R}^{2}$ of the symplectic bundle $\sigma^{*} \xi$ with symplectic form $\sigma^{*} d \lambda$. Here $\mathbb{R}^{2}$ is endowed with the standard symplectic form. We can use this trivialization to define a symplectic arc

$$
\Phi:[0, T] \rightarrow S p(1)
$$

by setting

$$
\Phi(t)=\psi\left(e^{2 \pi \mathrm{i} t / T}\right) \circ d \phi_{t}(x(0)) \circ \psi^{-1}(1) .
$$

Any such symplectic arc has a Conley-Zehnder index $\mu(\Phi)$, whose definition we recall below, and we define

$$
\mu(x, T)=\mu(\Phi) .
$$

It can be checked that $\mu(x, T)$ is well defined and does not depend on the choices made.

There are several possible ways to define $\mu(\Phi)$. We shall present the definition that is most appropriate to our purposes. Our reference for what follows is [9, Section 3].

Let $\Phi:[0, T] \rightarrow S p(1)$ be a smooth arc with $\Phi(0)=I$ and set $A(t):=-J \dot{\Phi}(t) \Phi(t)^{-1}$. Then $A(t)$ is a smooth path of symmetric 
matrices and $\Phi$ solves the linear differential equation:

$$
\dot{\Phi}=J A \Phi, \quad \Phi(0)=I
$$

for $t \in[0, T]$. Given $\tau \in \mathbb{R}$, suppose $v$ (not identically zero) solves the first order differential equation

$$
-J \dot{v}(t)-A(t) v(t)=\tau v(t)
$$

and satisfies the periodic boundary condition $v(0)=v(T)$. Then $v$ is an eigenvector with eigenvalue $\tau$ of the operator

$$
L_{A}(v)=-J \dot{v}-A(t) v
$$

defined on the space of $T$-periodic $H^{1}$-maps into $\mathbb{R}^{2}$. Since $v$ never vanishes we may choose a smooth angle $\varphi(t)$ such that

$$
e^{2 \pi \mathbf{i} \varphi(t)}=\frac{v(t)}{|v(t)|}, \quad t \in[0, T] .
$$

Define the winding number $\Delta(\tau, A) \in \mathbb{Z}$ by

$$
\Delta(\tau, A):=\varphi(T)-\varphi(0) .
$$

One can see that $\Delta(\tau, A)$ depends only on the eigenvalue $\tau$ and not on the eigenfunction $v$. For every integer $k \in \mathbb{Z}$ there are precisely two eigenvalues (counting multiplicities) $\tau_{1}$ and $\tau_{2}$ such that $k=\Delta\left(\tau_{1}, A\right)=$ $\Delta\left(\tau_{2}, A\right)$. Hence we may label the eigenvalues $\tau$ of $L_{A}$ by their winding numbers. Indeed, set $\Delta\left(\tau_{k}, A\right)=\left[\frac{k}{2}\right]$ for $k \in \mathbb{Z}$ and $\tau_{k} \leq \tau_{j}$ for $k \leq j$. With this labeling we define the Conley-Zehnder index of $\Phi$ as

$$
\mu(\Phi):=\max \left\{k: \tau_{k}<0\right\} .
$$

A contact form $\lambda$ on $M$ is said to be dynamically convex if $\mu(x, T) \geq 3$ for every contractible periodic solution of the Reeb vector field $X$. In [9] it is shown that if a contact form $\lambda$ on $\mathbb{S}^{3}$ comes from a strictly convex hypersurface in $\mathbb{R}^{4}$, then it is dynamically convex.

Recall from the previous sections that a Finsler metric on $\mathbb{S}^{2}$ defines a contact form in $\mathbb{S O}(3)$. We shall say that the Finsler metric is dynamically convex if its associated contact form is dynamically convex.

Given a Finsler metric on $\mathbb{S}^{2}$, let $\ell$ be the length of the shortest geodesic loop.

Theorem B. Let $F$ be a Finsler metric on $\mathbb{S}^{2}$ such that $K \geq \delta>0$. If $\ell>\pi / \sqrt{\delta}$, then $F$ is dynamically convex. In particular by the results in [9] any such Finsler metric has either two or infinitely many closed geodesics. 
Proof. By a simple rescaling argument we may assume that $K \geq 1$ and that $\ell>\pi$.

Consider a closed geodesic $\gamma$ with length $T$. The vector fields $X_{2}$ and $X_{3}$ provide a trivialization of $\xi$ without the need of extending the bundle to $D$. Given $\eta \in \xi$, write $\eta=a X_{2}+b X_{3}$. Then

$$
\eta \mapsto(b, a)
$$

gives a symplectic trivialization between $\left(\xi, d \omega_{1}\right)$ and $\mathbb{R}^{2}$ with the canonical symplectic form. (Note that we need to swap $a$ and $b$ since $d \omega_{1}=$ $-\omega_{2} \wedge \omega_{3}$.) Recall that if we write $d \phi_{t}(\eta)=x X_{2}+y X_{3}$, then $\dot{x}=y$ and $\ddot{x}+K x=0$. Thus, using the trivialization, we see that $\Phi$ satisfies the linear differential equation:

$$
\dot{\Phi}=J A \Phi
$$

where

$$
J=\left(\begin{array}{cc}
0 & -1 \\
1 & 0
\end{array}\right) \quad \text { and } A=\left(\begin{array}{cc}
1 & 0 \\
0 & K
\end{array}\right)
$$

Take $v$ an eigenvector of $L_{A}$ with eigenvalue $\tau$ and set $w(t):=\frac{v(t)}{|v(t)|}$. Using (14) it is straightforward to check that $w(t)$ satisfies

$$
\dot{w}=J(A+\tau I) w-w\langle w, J(A+\tau I) w\rangle .
$$

But $2 \pi \dot{\varphi} J w=\dot{w}$ and thus $2 \pi \dot{\varphi}=\langle(A+\tau I) w, w\rangle$. Since $K \geq 1$, we see that $\langle(A+\tau I) w, w\rangle \geq 1+\tau$ for all $t \in[0, T]$ and hence

$$
\Delta(\tau, A)=\varphi(T)-\varphi(0) \geq \frac{T}{2 \pi}(1+\tau) .
$$

Since $\Delta\left(\tau_{3}, A\right)=1$ we deduce

$$
\frac{T}{2 \pi}\left(1+\tau_{3}\right) \leq 1
$$

Let us combine this inequality with the lower bound on $\ell$. If $\gamma$ is a simple closed curve in $\mathbb{S}^{2}$, the orbit $t \mapsto x(t)=(\gamma(t), \dot{\gamma}(t))$ will not be contractible in $M$. If $\gamma$ is not a simple closed geodesic, then it must have length $T \geq 2 \ell>2 \pi$. Inequality (15) shows that $\tau_{3}<0$ and by the definition of $\mu$ we see that $\mu(x, T) \geq 3$ as desired.

Remark 6.1. The proof of the theorem indicates that one cannot expect to get dynamical convexity just assuming positive curvature. In fact if $\gamma$ is a closed geodesic with length $T$ such that $K=1$ along it, then the proof of the theorem shows that

$$
1=\frac{T}{2 \pi}\left(1+\tau_{3}\right) .
$$


Now consider a convex surface of revolution whose equator has $K=1$. It is clear that one can make the length $T$ of the equator as short as desired at the expense of increasing the curvature elsewhere. But using (16) we see that the Conley-Zehnder index of the equator iterated twice (to get a contractible curve in the unit sphere bundle) is $\leq 2$ if $T$ is small enough since $\tau_{3} \geq 0$ in this case. A concrete example of this is given by the ellipsoid in $\mathbb{R}^{3}$ :

$$
\frac{x^{2}+y^{2}}{a^{2}}+z^{2}=1
$$

The equator $z=0$ has curvature $K=1$ and length $2 \pi a$, so for $a=1 / 2$ we get that the equator iterated twice has $\tau_{3}=0$ and $\mu=1$. Note that in this case $1 \leq K \leq 16$. This example shows that Theorem $\mathrm{B}$ is in fact sharp.

Hence there are positively curved Riemannian metrics on $\mathbb{S}^{2}$ such that under the lifting procedure described in Lemma 4.1 they do not give rise to convex hypersurfaces in $\mathbb{R}^{4}$.

The results of Hofer, Wysocki and Zhender in [9] treat a lot more than just the dichotomy "two or infinitely many" closed orbits. Their results state that the Reeb flow has a closed disk as a surface of section whose boundary is an unknotted closed orbit $P$ with $\mu=3$. Moreover, any other closed orbit has to be linked with $P$.

If the Finsler metric is reversible, then one can obtain a lower bound for $\ell$ from an upper bound on curvature. Indeed if $0<K<a$, then $\ell>2 \pi / \sqrt{a}$. For Riemannian metrics this is a classical result (cf. [12, Theorem 3.4.8]) and for reversible Finsler metrics the proof is quite the same (cf. [14, Theorem 4]).

Thus Theorem B implies:

Corollary 6.1. A strictly 1/4-pinched positively curved reversible Finsler metric is dynamically convex.

The corollary seems new even for the case of Riemannian metrics. Given a Finsler metric $F$, the reversibility $r$ of $F$ is (cf. [14]):

$$
r:=\max _{(x, v) \in M}\{F(x,-v)\} \geq 1 .
$$

Clearly $r=1$ iff $F$ is reversible. In [14, Theorem 4] Rademacher gives a lower bound for the length $L$ of the shortest closed geodesic as follows. If $0<K \leq 1$, then

$$
L \geq \pi(1+1 / r)
$$

The same bound holds for $\ell$ [15] and hence we may also conclude that a Finsler metric with $\left(1-\frac{1}{1+r}\right)^{2} \leq K<1$ is dynamically convex. 


\section{REFERENCES}

[1] V. Bangert, Y. Long, The existence of two closed geodesics on every Finsler 2-sphere, preprint.

[2] D. Bao, S.S. Chern, Z. Shen, An introduction to Riemann-Finsler geometry, Graduate Texts in Mathematics, 200. Springer-Verlag, New York, 2000.

[3] D. Bao, David, C. Robles, Z. Shen, Zermelo navigation on Riemannian manifolds, J. Differential Geom. 66 (2004) 377-435.

[4] R. Bryant, Projectively flat Finsler 2-spheres of constant curvature, Selecta Math. 3 (1997) 161-204.

[5] R. Bryant, Some remarks on Finsler manifolds with constant flag curvature, Houston J. Math. 28 (2002) 221-262.

[6] R. Bryant, Geodesically reversible Finsler 2-spheres of constant curvature, preprint, math.DG/0407514.

[7] G. Contreras, F. Oliveira, $C^{2}$ densely the 2-sphere has an elliptic closed geodesic, Ergod. Th. and Dynam. Sys. 24 (2004) 1395-1423.

[8] A. Harris, K. Wysocki, Branch structure of J-holomorphic curves near periodic orbits of a contact manifold, Trans. Am. Math. Soc. to appear (cf. also math.CV/0701496)

[9] H. Hofer, K. Wysocki, E. Zehnder, The dynamics of three-dimensional strictly convex energy surfaces, Ann. of Math. 148 (1998) 197-289.

[10] H. Hofer, K. Wysocki, E. Zehnder, Finite energy foliations of tight threespheres and Hamiltonian dynamics, Ann. of Math. 157 (2003) 125-255.

[11] A. Katok, Ergodic properties of degenerate integrable systems, Math. USSR Izv. 7 (1973) 535-572.

[12] W. Klingenberg, Riemannian geometry, De Gruyter Studies Math.1, de Gruyter, Berlin-New York, 1995.

[13] G.P. Paternain, Geodesic flows, Progress in Mathematics, 180 Birkäuser 1999.

[14] H.B. Rademacher, A sphere theorem for non-reversible Finsler metrics, Math. Ann. 328 (2004) 373-387.

[15] H.B. Rademacher, Personal communication.

[16] Z. Shen, Two-dimensional Finsler metrics with constant curvature, Manusc. Math. 109 (2002) 349-366.

[17] J.C. Sikorav, Singularities of J-holomorphic curves, Math. Z. 226 (1997) 359373.

[18] W. Ziller, Geometry of the Katok examples, Ergod. Th. and Dynam. Sys. 3 (1983) 135-157.

School of Mathematics, Statistics and Computer Sciences, UniverSity of New England, Armidale NSW 2351, Australia

E-mail address: adamh@turing.une.edu.au

Department of Pure Mathematics and Mathematical Statistics, University of Cambridge, Cambridge CB3 0WB, England

E-mail address: g.p.paternain@dpmms.cam.ac.uk 